\title{
AN APPLICATION OF INTERNET OF THINGS IN THE FIELD OF URBAN BUILDING FIRE SAFETY
}

\author{
KE YIN \& JUNCHENG JIANG \\ College of Urban Construction and Safety Engineering, \\ Nanjing University of Technology, Nanjing Jiangsu 210009, China.
}

\begin{abstract}
The Internet of Things (IoT) has become an important part in day-to-day activities, and it has become one of the important methods used in the development of information network technology. In this paper, the IoT technology is applied in buildings that are on fire to create a dynamic model of analysis based on relevant location algorithm. Compared with the traditional models of fire control systems, it has been found that the IoT-based models have at least three advantages: (1) can make a more timely collection of information; (2) can analyze information dynamically; and (3) can provide the best rescue plan, which is useful to establish an information fire control system. The application of this system can reduce the loss of property and the risk of personnel danger, providing an important reference for the construction of information system for fire protection and scientific management.

Keywords: Algorithm, IoT, urban building, fire safety.
\end{abstract}

\section{INTRODUCTION}

Recently, with the development and reformation of the state's economy, city construction has drawn much attention from both government and society, especially the construction plan of Smart City in China. So it is quite natural to pay more attention to urban public security in this construction based on Internet of Things (IoT). It is known that the foundation of Urban Public Safety Emergency Management is very weak in China, especially fire safety. Big fire accidents lead to a lot of loss of people's life and assets. For instance, a series of recent incidents such as the Shanghai JingAn Fire and the Nanjing Chemical Plant Explosion caused great losses. How to prevent and deal with urban public safety incidents has become a very urgent topic worth widely exploring. Based on previous researches, here the writer wants to put forward a plan of using IoT technology with certain algorithm to construct a practical model of monitoring fire in urban buildings.

\section{A BRIEF SURVEY OF IOT}

On December 8, 2011, the Ministry of Industry and Information Technology of the People's Republic of China released the 12th Five-Year Plan on IoT, of which seven main tasks involved 'the construction of standard systems of IoT,' and 'the demonstration of active application of IoT.' Against the background of the national strategies of 'Sense China', then the IoT has successfully been transformed from a concept to an industry. According to ITU Internet Reports 2005 [1], The Internet of Things is the extension of internet applications, which mainly consists of four concepts: 'Radio Frequency Identification (RFID), Wireless Sensor Networks (WSN), intelligent technology and nanotechnology.' These four kinds of core technologies play an important role in each field in controlling the fire on buildings. Among them, RFID is the foundation and networking core of the construction of IoT [2].

It is known that there are various kinds of RFID and WSN development of network protocols such as ZigBee [3], 6LoWPAN [4], and Ultra Wideband (UWB), of which Shen Xuesong et al. [5] applied WSN for resource tracking in building construction and analyzed different network protocols in detail. 
IoT has become the inevitable product of rapid development of intelligent technology, which appropriately meets the needs of intelligent terminal development. It is known that intelligent technology is widely used through embedded technology with intellectual systems embedded in the objects to make them smart. These smart detectors can flexibly switch mode according to the threshold, providing more accurate warning information while reducing energy consumption.

With the development of microminiaturization design of various sensing devices, the carrier that stores all kinds of data and information is miniaturized so that it can be easily installed in a variety of objects without affecting its normal operation and circulation. The use of nanotechnology implies that the IoT technology can be used in a smaller volume of fire-fighting equipment, for example, the active RFID tag, which is the successful combination of intelligent technology and nanotechnology.

\section{PREVIOUS MODELS OF BUILDING FIRE SAFETY}

IoT has three outstanding characteristics: overall perception, reliable transmission, and intelligent processing. It is also known as a sensor network, which refers to a vast network by means of combining various information-sensing devices such as RFID, infrared sensors, Global Positioning System (GPS), laser scanners, and others with an Internet connection [6]. These IoT characteristics can properly meet the three basic requirements in the field of fire safety management of fire control, fire alarm, and fire rescue, respectively. However, there are only a few reports on making full use of the three functions of IoT together in urban building fire safety.

Generally, building fire warning and monitoring system can be divided into two types, one is based on static model stimulation analyzing the spread trend of fire and smoke and people's movements inside the building. Chu Yanyan et al. [7], based on the analysis of the inner building, adapted the combination of In-house Fire Evacuation and Fire Dynamic Simulator to simulate the smoke controlling and the evacuation of trapped people. They got certain strategies to control fire and smoke from the investigation. However, they failed to provide the practical monitoring and urgent regulating strategies. The other system involved the IoT application approach in the public fire protection field with the main focus on monitoring, i.e. Damian Gallegos from the University of New Mexico applied wireless sensor technology to forest fire protection, and established an effective early warning and fire monitoring system [8]. Stanford University developed a building monitoring system based on a WSN for monitoring, as well as periodic test of fire accidents [9]. Some equipments inside the building (such as an elevator) and outside the building (such as fire extinguishers and fire alarm equipments) have also been introduced to the concept of IoT [10]. Uwe Rueppel [11] constructed a Building Information Modeling system based on IoT technology for evacuating people from dangerous places inside the building. These researches have often been cited as the successful cases in urban safety. But these researches either paid more attention to the ideal settings or focused on certain specific algorithm, lacking overall analysis to the whole setting. Therefore, it is quite necessary to create a new way to explore feasibility of IoT application in the whole process of urban fire protection and fire safety.

\section{APPLICATION OF IOT IN URBAN BUILDING FIRE SAFETY}

IoT is multidisciplinary, i.e. involves many research areas such as hardware, short distance data transmission, network, data fusion, accident decision tools, and so on. Therefore, it is quite realistic to apply IoT in the field of building fire, that is, to apply IoT to integrate all kinds of technologies and then put them into specific work of each stage in the fire. With this 
intention, the writer discusses building fire protection process in two stages based on the application of the key technologies of the IoT: intelligent management and intelligent command. Here, by the intelligent management it means prevention and early warning of building fire and by intelligent command, the emergency rescue and command during and after fire.

\subsection{IoT-based intelligent management of building fire safety system}

As discussed above, IoT can be widely used in building fire systems, especially in the construction of fire equipment and personnel density monitoring models. According to the analysis of the causes of fire in high-rise buildings, several common problems can easily be found: (1) how to deal with the lack of effective management of fire-fighting equipment and supervision in daily maintenance after initial acceptance in building; (2) how to obtain parameters of key areas and danger sources accurately and timely; and (3) how to find emergency rescue methods in a crowded place.

Since the RFID indoor locating technology is the key point of indoor active surveillance application of IoT, people usually use RFID in their development of problem-solving project, mainly adopting the SpotoN model and 3D-iD indoor locating technology based on Radio Frequency (RF), which is similar to SpotoN using wireless local network technology. These two kinds of technologies are of high reliability, but the investment in the system is often too large. Lionel et al. used LocaAtioN identification based on dynamic Active Rfid Calibration (LANDMARC) model [12] to introduce the reference tags which reduce the need to use RFID readers, and according to the residual weighting algorithm between different labels, a higher location accuracy can be obtained. This model is considered to be the active dynamic positioning recognition technology with a better performance (see Fig. 1).

According to the model (Fig. 1), sensors continuously read information transmitted from RFID tags, and the received signal strength is defined as Signal Strength Vector. Since the tags are classified with tracking tags and reference tags, the Signal Strength Vector of a tracking tag is represented as $S=\left(S_{1}, S_{2}, \ldots, S_{n}\right)$, where $S_{i}$ denotes the signal strength of the tracking tag perceived on reader $i$, where $I=1, \ldots, n$. The Signal Strength Vector of a reference tag is denoted as $\theta=\left(\theta_{1}, \theta_{2}, \ldots, \theta_{n}\right)$, where $\theta_{i}$ is the signal strength of the reference tag perceived on

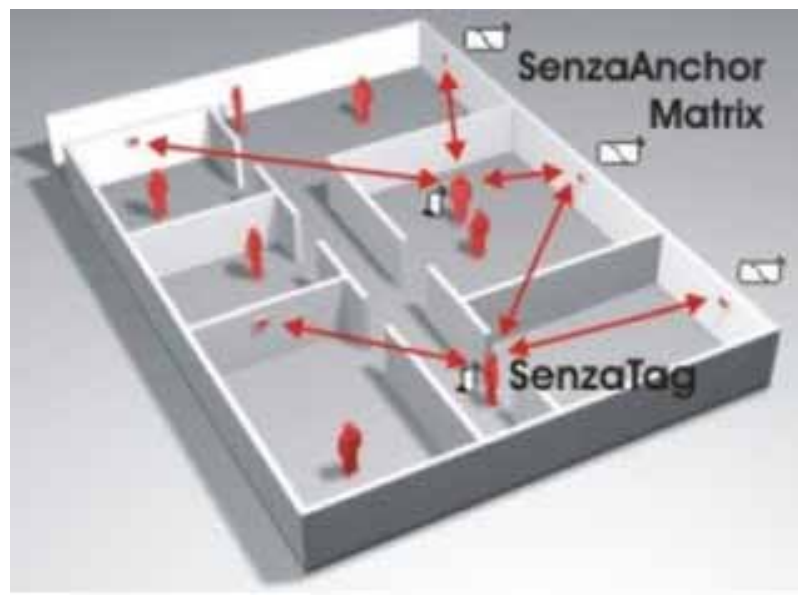

Figure 1: LANDMARC model. 
reader $i$, where $i=1, \ldots, n$. Each RFID reader will receive the two types of data, and for each individual tracking tag $p$, where $p=1, \ldots, u$, the following formula applies

$$
E_{u j}=\sqrt{\sum_{i=1}^{n}\left(\theta_{j i}-S_{u i}\right)^{2}} j \in(1, m)
$$

$E_{u j}$ is the Euclidean distance in signal strength between a tracking tag and a reference tag $r_{j}$. $E_{u j}$ represents the location relationship between the reference tags and the tracking tag. If there are $m$ reference tags, a tracking tag will have its $E$ vector as $E=\left(E_{1}, E_{2}, \ldots, E_{m}\right)$. Therefore, the reference tag closer to the tracking tag is supposed to have a smaller $E$ value [13].

The weights assigned to different neighbors:

$$
w_{j}=\frac{1 / E_{i}^{2}}{\sum_{i=1}^{k} 1 / E_{i}^{2}}
$$

$w_{j}$ is the weighting factor to the $i$ th neighboring reference tag.

After the weight being obtained, the computed tracking tag location $(x, y)$ is given by,

$$
(x, y)=\sum_{i=1}^{k} w_{i}\left(x_{i}, y_{i}\right)
$$

at the end, the error distance is used to check the LANDMARC system. The error value can be obtained as the difference between the computed coordinates $(x, y)$ and the real coordinates $\left(x_{0}, y_{0}\right)$, so as to improve the correlated parameters.

Fire safety hazards located inside the building are inevitable if they depend only on the longterm artificial check-out, and what's more, the accuracy of fire equipment requires a higher professional level in terms of persistence and reliability [14]. Once the equipment fault occurs, the network system can find it in time and inform the relevant department that takes care of its maintenance. The equipment manufacturers can also trace the location of the faulty device and make the necessary repairs. Aside from its use in the daily management of fire protection systems, the LANDMARC model is able to obtain accurate information regarding the use of fire equipment or to locate the ignition position on time. This is especially useful when facing dense flow in the building where LANDMARC not only monitors the real-time flow density but also provides some simulation and verification, by asking the relevant personnel to wear the embedded RFID tags and comparing them with the indoor fixed labels so as to guide them in an orderly and effective manner. Therefore, the application of IoT in fire safety management inside the building can not only play an effective monitoring role in the fire-fighting equipment maintenance but also provide early warning of dangerous areas to unsuspecting people.

\subsection{IoT-based fire-fighting intelligent command and emergency rescue}

Fire accidents are unexpected in a building, and especially, the complexity of structures between layers in high-rise buildings will increase the difficulty of fire fighting. When fire occurs, the fire spreads fast, usually due to a chimney effect, so it is quite necessary to improve the efficiency of fire emergency rescue. However, rescue operations in buildings that are on fire are often confronted with many difficulties such as: (1) The fire spreads quickly and the different vertical wells, such as elevator wells, tube wells, air ducts, cable wells, and stairways in high-rise buildings, often make personnel rescue resort to personal experience, which is often accompanied by lack of communication with the outside world and the lack of a uniform dispatch and control. This can lead to ineffective rescue operations, and an increase 
in risk due to lack of information about the latest state of the fire. (2) The evacuation difficulty, which indicates that the number of people is more in high-rise buildings; also, it is difficult to accurately know the distribution of people and the flow condition within the building. (3) The difficulty in deciding the routes of rescue vehicles. Since traffic in modern city streets is often jammed and the routes between buildings are quite complex, rescue vehicles often cannot get near the fire in time, which leads to delays in the rescue.

Of course, GPS technology can provide help for the rescue vehicle location and route location, but it is only suitable for outdoors. Here, IoT helps a lot in fire fighting and rescue by combining outdoor GPS systems with indoor real-time location systems. The indoor location system mainly includes Real-Time Location Systems (RTLS) if the position is updated within an interval of one minute [15] based on ISO/IEC 24730 systems for position sensing. RTLS works mainly on WSN technology to collect data through the indoor positioning algorithm to build a dynamic analysis model. Position sensing via RF is based on the calculation of the distance between the sender and the receiver (antenna). Normal RF RTLS have to cope with overlapping signals of the direct signal and signals reflected from walls, windows, etc. (multipath effect). The advantage of UWB is the use of very short pulses, which are possible because of a wide range of frequencies [11].

In indoor fire rescue, such parameters as the concentration of harmful gases including carbon dioxide, the air humidity, the flame trend, and the location of indoor personnel and rescue workers will be collected into a relevant RF module, which will be sent to the analysis module of the host computer by the set-up WSN based on UWB technology. The indoor localization algorithm based on UWB technology primarily includes four different measurement principles: Angle of Arriva, Received Signal Strength Indication, Time of Arrival (TOA), and Time Difference of Arrival.

Based on the above discussion, a data location model is designed to set up a monitoring network including the trapped people inside and outside the building on fire, the fire rescue personnel, and the positioning of the vehicles (see Fig. 2).

The network model (Fig. 2) consists of fixed nodes, mobile nodes, and control nodes. The fixed nodes refer to the set sensor nodes embedded inside the building in advance; the mobile

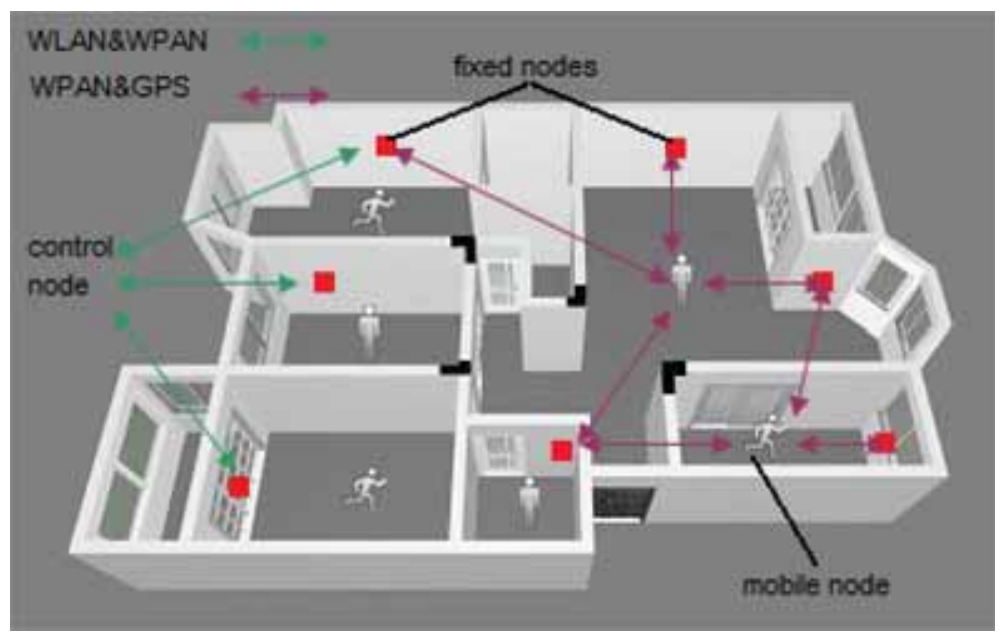

Figure 2: UWB-based net model. 
nodes are attached to the trapped personnel and the rescuers, including RFID tags and GPS communication equipments. The control nodes are usually used to collect all the information of personnel and related equipment. Thus, the monitoring network works effectively by comparing information from the fixed nodes with that of mobile nodes, while the fixed nodes, as reference nodes at the same time, transfer the information of the temperature and environment in the relevant area to the control nodes in real time. Due to the adoption of UWB technology, the information from mobile nodes can be efficiently transmitted to the control nodes. By excluding the interference from the distress site, the mobile nodes can directly reflect the staff's temperature, pulse, and the parameters of the surrounding air. At the same time, based on the information from fixed nodes, the control nodes will analyze the data and information and then construct a three-dimensional model.

By means of the TOA algorithm [16], the UWB locating model can be divided into two parts: in the first part, the time-delay of UWB signal will be estimated, by using the third-order cumulants [17] to get rid of the sensitivity of Nearly Gaussian Noise, with two assumed measuring data $\left(x_{1}(n)\right.$ and $\left.x_{2}(n)\right)$ as follows:

$$
\left\{\begin{array}{l}
x_{1}(n)=s(n)+w_{1}(n) \\
x_{2}(n)=s(n-D)+w_{2}(n)
\end{array}\right.
$$

In the above formula, $s(n-D)$ stands for the time-delay signal of $s(n), D$ for the time-delay, $w_{1}(n)$ and $w_{2}(n)$ refer to Gaussian noise, which are all independent to $s(n)$, and $x_{1}(n)$ and $x_{2}(n)$ will be calculated together with their relevant cumulants. Finally, by testing the spectral peak, the value of time-delay of the signal can be estimated. In the second part, the spherical location model for TOA was adapted. This model needs at least four reference nodes to set up four equations. Suppose the reference node $i$ has coordinates in $\left(x_{i}, y_{i}, z_{i}\right)$, and the target node has coordinates in $(x, y, z)$. Then, based on the distance between every reference node and every target node, four equations can be obtained:

$$
\sqrt{\left(x-x_{i}\right)^{2}+\left(y-y_{i}\right)^{2}+\left(z-z_{i}\right)^{2}}=c t_{i} \quad i=1,2,3,4
$$

Here, $c$ stands for the light speed and $t_{i}$ for the time of transmitting the signal to the $i$ reference node, i.e. the value of TOA time-delay.

$x, y, z$ can be easily calculated by the geometric method of non-iterative algorithm to obtain the coordinates of the to-be-located target nodes [18]. Location and position of the trapped personnel and the moving route can be calculated effectively.

Thus, a WSN area can be built both inside and outside the building on fire, and the commanding personnel can judge the spread of the fire inside the building through the rescue personnel in the fire spot and take direct and effective measures. More important is that they can receive information about the location and physical status of the trapped persons and rescue personnel, from this, they can make reasonable planning of rescue and evacuation routes in real time so as to guarantee the safety of the trapped personnel.

\section{CONCLUSIONS}

The latest trend for developing information-based systems is based on the IoT. In this paper an IoT-based model is suggested to help control building fires and improve fire evacuation and rescue. Compared with traditional models of fire control systems, it has been found that the IoT-based models discussed above have at least three advantages: (1) can make a more timely collection of information; (2) can analyze the information dynamically; and (3) can provide best rescue plan; which is useful to establish an information fire control 
system. The application of this system can reduce the loss of property and the risk of personnel danger.

However, if people want to get more effective methods to supervise the fire inside high-rise buildings, they have to consider the following (1) how to use a sensor in a dark environment to obtain the parameters of temperature, pressure, and air concentration and then analyze the disaster so as to simulate the disaster development online; (2) how to exclude interference such as indoor noise, high temperature and make full use of the WSNs to locate and monitor the rescuers, in order to make the information fire control system more effective.

\section{ACKNOWLEDGEMENTS}

Yin Ke acknowledges the support of Jiangsu Graduate Scientific Innovation Projects. The authors also acknowledge two anonymous reviewers for their suggestions to revise the manuscript.

\section{REFERENCES}

[1] International Telecommunication Union (ITU), ITU Internet Reports 2005: the Internet of Things. Tunis: World Summit on the Information Society (WSIS), pp. 17-18, 2005.

[2] Baoan Li \& Jianjun Yu, Research and application on the smart home based on component technologies and Internet of Things. Procedia Engineering, 15, pp. 2087-2092, 2011. doi: http://dx.doi.org/10.1016/j.proeng.2011.08.390

[3] ZigBee Alliance, Electronic Text at, http://www.zigbee.org (accessed February 2013).

[4] IETF, IPv6 over IEEE 802.15.4 low-power wireless personal-area-network. Electronic Text at, http://www.6lowpan.org 2007 (accessed February 2013).

[5] Shen Xuesong \& Chen Wu, Wireless sensor networks for resources tracking at building construction sites. Tsinghua Science and Technology, 13(S1), pp. 78-83, 2008.

[6] Tu Qiang \& Wang Lei, Analysis and prospect about application of IoT in super highrise building. Modern Architecture Electric, 1(7), pp. 1-3, 2010.

[7] Chu Yanyan, Zhang Hui \& Yang Rui, Building fire smoke control strategies simulation using fire and evacuation information. Journal of Tsinghua University (Science \& Technology), 50(8), pp. 1158-1162, 2010.

[8] Damian Gallegos, Enhanced logistical information in fire combat post 911. Master Thesis. Summer Undergraduate Program in Engineering Research at Berkeley SUPERB, Sep 2003.

[9] Du Chunquan \& Zhu Shunbing, Research on urban public safety emergency management early warning system based on technologies for the Internet of Things. Procedia Engineering, 45, pp. 748-754, 2012. doi: http://dx.doi.org/10.1016/j.proeng.2012.08.234

[10] Zhu Shunbing, Wei Qiuping \& Du Chunquan, J., Study and prospect on the application of Internet of Things in perceiving safety. China Safety Science Journal, 20(11), pp. 164-170, 2010.

[11] Rueppel, U. \& Stuebbe, K.M., BIM-Based indoor-emergency-navigation-system for complex buildings. Tsinghua Science and Technology, 13(S1), pp. 362-367, 2008. doi: http://dx.doi.org/10.1016/S1007-0214(08)70175-5

[12] Lionel, M.N., Yunhao, L. \& Yiu, C., LANDMARC: indoor location sensing using active RFID $[\mathrm{C}]$. Proceedings of the First IEEE International Conference, Vol. 10, pp. 407-415, 2003.

[13] Guo Zijian, Study on LANDMARC indoor location system. Master Thesis, National Taiwan University of Science and Technology, 2009. 
[14] Zhang Ying-cong \& Yu Jing, A study on the fire IoT development strategy. Procedia Engineering, 52, pp. 314-319, 2013. doi: http://dx.doi.org/10.1016/j.proeng.2013.02.146

[15] Uckelmann, D. \& Hofmann, S., RTLS-real time location systems. SMART Solutions 2008. rfid ready Verlag, Isernhagen, Germany, 2008.

[16] Cui Xue-rong, Zhang Hao \& Wu Chun-lei, A study on the simulations of location algorithm for the nodes in UWB-based Internet of Things. Science Technology and Engineering, 11(27), pp. 6621-6625, 2011.

[17] $\mathrm{Wu}, \mathrm{Y}$., Time delay estimation of non-Gaussian signal in unknown Gaussian noises using third-order cumulants. Electronics Letters, 38(16), pp. 930-931, 2002. doi: http:// dx.doi.org/10.1049/el:20020584

[18] Liang Jiuzheng, Wireless Location System. Publishing House of Electronics Industry: Beijing, China, 2013. 